\title{
Maximal pericoronary adipose tissue thickness is associated with hypertension in nonobese patients with acute or chronic illness
}

\author{
Shinhae Chang ${ }^{1}$, Wangjin Lee ${ }^{1}$, Byounggyu Kwag ${ }^{1}$, Yun Hee Chung ${ }^{2}$, and In Sook Kang ${ }^{3,4}$
}

\begin{abstract}
${ }^{1}$ Department of Family Medicine, Green Hospital, Seoul; Departments of ${ }^{2}$ Family Medicine and ${ }^{3}$ Internal Medicine, Gimpo Woori Hospital, Gimpo; ${ }^{4}$ Cardiovascular Research Institute, Yonsei University College of Medicine, Seoul, Korea
\end{abstract}

Background/Aims: Recent studies have shown an association of epicardial fat thickness with diabetes and hypertension (HTN) in asymptomatic populations. However, there is lack of information as to whether there is similar association between pericoronary adipose tissue (PAT) and HTN in the patients who have acute or chronic illness.

Methods: This study included 214 nonobese patients hospitalized with acute or chronic noncardiogenic illness. PAT thicknesses were measured from fat tissues surrounding left and right coronary arteries in enhanced, chest computed tomography scans, yielding the maximal PAT value from left and right coronary arteries was used for analysis. Baseline data from hypertensive $(n=81)$ and normotensive $(n=133)$ patients were collected and compared.

Results: PAT is positively correlated with age $(r=0.377, p<0.001)$, body mass index (BMI; $r=0.305, p<0.001)$, systolic blood pressure $(r=0.216, p=0.001)$, and total cholesterol $(r=0.200, p=0.006)$. The hypertensive group was older $(69.58 \pm 11.69$ years vs. $60.29 \pm 14.98$ years), and had higher PAT content $(16.30 \pm 5.37 \mathrm{~mm}$ vs. 13.06 $\pm 5.58 \mathrm{~mm})$ and BMI $\left(23.14 \pm 3.32 \mathrm{~kg} / \mathrm{m}^{2}\right.$ vs. $\left.20.96 \pm 3.28 \mathrm{~kg} / \mathrm{m}^{2}\right)$ than the normotensive group (all $p<0.001$ ). Multivariate analysis showed that age (odds ratio [OR], 2.193; $p=0.016)$, PAT thickness (OR, 1.065; $p=0.041)$, and BMI $\left(25 \leq \mathrm{BMI}<30 \mathrm{~kg} / \mathrm{m}^{2}\right.$; OR, 6.077; $p=0.001$ ) were independent risk factors for HTN.

Conclusions: In nonobese patients with noncardiogenic acute or chronic illness, PAT thickness is independently correlated with HTN, age, and BMI.

Keywords: Adipose tissue; Hypertension; Multidetector computed tomography; Coronary vessels

\section{INTRODUCTION}

Epicardial adipose tissue (EAT) is a special type of fat deposited within the epicardium and wrapped around the myocardial tissue and coronary arteries $[1,2]$. This fat depot is not separated from the underlying tissue by fascia, and releases a wide range of biologically active molecules that modulate vascular smooth muscle contraction $[1,3]$. EAT is considered to play a significant role in the pathogenesis of coronary artery disease (CAD) through local release of endocrine and paracrine factors $[1,3,4]$.

EAT can be further divided into myocardial adipose tissue (MAT) over the myocardium, and pericoronary adipose tissue (PAT) surrounding the major coronary arteries. Although anatomically contiguous, MAT and 
PAT showed distinct responses to aerobic exercise training in the pig model [2]. Because PAT directly covers the surface of coronary arteries, it may have the most immediate impact on physiological and pathological processes of the coronary arteries [5-7], and may play an important role in cardiovascular disease and metabolic syndrome $[8,9]$.

Several studies have reported EAT to be associated with coronary artery obstructive disease, coronary calcification, atherosclerosis, and atrial fibrillation (AF) $[3,6,8,10,11]$. Hypertension (HTN) is one of the most important, modifiable risk factors for heart disease and is also a component of metabolic syndrome, akin to dyslipidemia, obesity, and insulin resistance [12,13]. A few studies have investigated this topic, suggesting that increased EAT may be associated with HTN in asymptomatic patients $[14,15]$. Most studies assessed EAT rather than PAT per se, and questions remain, as to whether there is an association between EAT and HTN in nonobese individuals in the populations in acute or chronic illness. Hence, we conducted this study to examine the relationship between PAT thickness and HTN in nonobese patients hospitalized for acute or chronic noncardiogenic illnesses.

\section{METHODS}

\section{Study population}

Patients hospitalized due to noncardiac acute or chronic illnesses underwent enhanced chest computed tomography (CT) between January 2014 and September 2015, as part of a screening procedure for lung disease. This is a cross-sectional study and medical records were retrospectively reviewed. Out of an initial group of $238 \mathrm{pa}-$ tients, data from 214 were included in this study after excluding 24 patients based on (1) poor CT image quality (five subjects); (2) a history of either heart disease, chronic kidney disease, thyroid disorders, alcohol or drug abuse ( 16 subjects); ( 3 ) a body mass index (BMI) $\geq 30 \mathrm{~kg} /$ $\mathrm{m}^{2}$ (one subject); and (4) unavailability of medical information (two subjects).

Patients were variously admitted to different departments as follows: internal medicine ( $\mathrm{n}=103,48.13 \%)$, general surgery ( $\mathrm{n}=17,7.94 \%)$, occupational medicine $(n=63,29.44 \%)$, neurology $(n=2,0.47 \%)$, orthopedic sur- gery $(n=12,5.61 \%)$, neurosurgery $(n=16,7.48 \%)$, and rehabilitation medicine $(\mathrm{n}=1,0.47 \%)$.

\section{Data collections and definitions}

Study data were collected from medical records describing previous medical history and laboratory measurements. Participants were considered as current smokers if they reported having smoked within the last 6 months. Baseline parameters including patients' heights, weights and blood pressures were obtained from medical records. HTN was defined as systolic blood pressure (SBP) $>140 \mathrm{mmHg}$, diastolic blood pressure (DBP) > $90 \mathrm{mmHg}$, or current use of antihypertensive medications. Diabetes was defined as a fasting blood glucose level of $>126 \mathrm{mg} /$ $\mathrm{dL}$, a glycated hemoglobin value $>6.5 \%$, or the current use of oral hypoglycemic agents or insulin. AF was detected by electrocardiography upon admission.

Obesity was defined as a BMI $\geq 30 \mathrm{~kg} / \mathrm{m}^{2}$. Participants were categorized into three subgroups: underweight, BMI < $18.5 \mathrm{~kg} / \mathrm{m}^{2}$; normal, $18.5 \leq \mathrm{BMI}<25 \mathrm{~kg} / \mathrm{m}^{2}$; and overweight, $25 \leq \mathrm{BMI}<30 \mathrm{~kg} / \mathrm{m}^{2}[16]$.

\section{Pericoronary adipose tissue thickness measurement} In this study, multidetector computed tomography (MDCT) scans were taken using a 16 channel, multidetector scanner (Brightspeed Elite Definition 16, General Electric, Chicago, IL, USA). Each scan was obtained during a single breath hold. Slice thickness was $3 \mathrm{~mm}$ and following an injection of $100 \mathrm{cc}$ of contrast medium ( $2 \mathrm{cc} / \mathrm{sec}$ ), average scan times were as follows: scout views for 2 seconds at $120 \mathrm{kVp}, 10 \mathrm{~mA}$; pre-contrast imaging for 10 seconds at $120 \mathrm{kVp}, 200$ to $230 \mathrm{~mA}$; and postcontrast imaging for 7.95 seconds at $120 \mathrm{kVp}, 200$ to $230 \mathrm{~mA}$. Maximal PAT thickness around the left coronary artery (LCA) and right coronary artery (RCA) was measured along the longest, continual mass of PAT around the proximal portion of each artery. Since the thickness of a single slice image was $3 \mathrm{~mm}$, PAT measurements were obtained by examining approximately 10 slices. PAT measurement along the LCA and RCA is described in Fig. 1. All measurements were conducted by a physician blinded to patient medical information, and confirmed thereafter by a cardiologist.

\section{Statistical analysis}

Baseline characteristics of subjects were compared us- 
Table 1. Baseline characteristics of the study population $(\mathbf{n}=\mathbf{2 1 4})$

\begin{tabular}{|c|c|c|c|c|}
\hline Variable & Overall $(n=214)$ & Hypertension $(n=81)$ & Normotension $(n=133)$ & $p$ value \\
\hline Male sex & $152(71.0)$ & $53(65.4)$ & $99(74.4)$ & 0.159 \\
\hline Age, yr & $63.80 \pm 14.49$ & $69.58 \pm 11.69$ & $60.29 \pm 14.98$ & $<0.001$ \\
\hline Smoker & $50(23.4)$ & $14(17 \cdot 3)$ & $36(27.1)$ & 0.101 \\
\hline Diabetes mellitus & $43(20.1)$ & $20(24.7)$ & $23(17 \cdot 3)$ & 0.190 \\
\hline Atrial fibrillation & $10(4.7)$ & $5(6.2)$ & $5(3.8)$ & 0.417 \\
\hline Body mass index, kg/m² & $21.76 \pm 3.47$ & $23.14 \pm 3.32$ & $20.96 \pm 3.28$ & $<0.001$ \\
\hline $\mathrm{SBP}, \mathrm{mmHg}$ & $124.16 \pm 18.59$ & $132.26 \pm 18.01$ & $119.11 \pm 17.18$ & $<0.001$ \\
\hline $\mathrm{DBP}, \mathrm{mmHg}$ & $76.64 \pm 10.97$ & $79 \cdot 53 \pm 10.17$ & $74.86 \pm 11.13$ & 0.002 \\
\hline $\mathrm{AST}, \mathrm{IU} / \mathrm{L}$ & $38.91 \pm 46.35$ & $42.48 \pm 62.57$ & $36.81 \pm 33.00$ & 0.388 \\
\hline ALT, IU/L & $29.19 \pm 40.52$ & $28.54 \pm 28.17$ & $29.64 \pm 46.74$ & 0.849 \\
\hline GGT, IU/L & $65.66 \pm 90.86$ & $65.89 \pm 101.59$ & $65.97 \pm 84.69$ & 0.995 \\
\hline Total cholesterol, mg/dL & $156.17 \pm 46.89$ & $156.25 \pm 51.38$ & $156.45 \pm 44.28$ & 0.977 \\
\hline Triglyceride, mg/dL & $126.36 \pm 82.15$ & $129.51 \pm 79.46$ & $123.68 \pm 84.13$ & 0.660 \\
\hline Fasting glucose, $\mathrm{md} / \mathrm{dL}$ & $123.84 \pm 45.85$ & $124.97 \pm 43.44$ & $123.43 \pm 47.48$ & 0.816 \\
\hline Maximal PAT thickness, mm & $14.25 \pm 5.73$ & $16.30 \pm 5.37$ & $13.06 \pm 5.58$ & $<0.001$ \\
\hline
\end{tabular}

Values are presented as number (\%) or mean \pm SD.

SBP, systolic blood pressure; DBP, diastolic blood pressure; AST, aspartate transaminase; ALT, alanine aminotransferase; GGT, $\gamma$-glutamyl transferase; PAT, pericoronary adipose tissue.

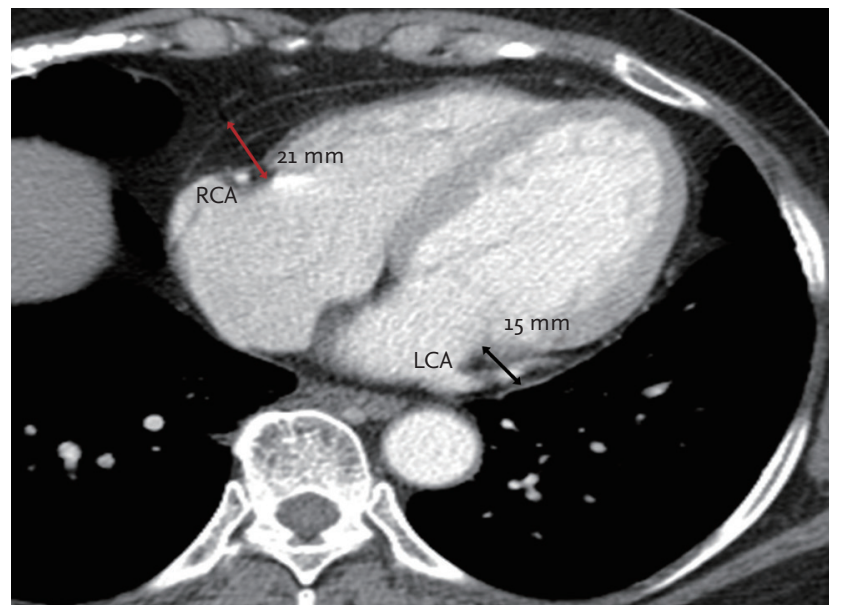

Figure 1. Measurement of pericoronary adipose tissue (PAT) thickness. The red arrow indicates the longest PAT thickness on surrounding proximal portion of right coronary artery (RCA) and the black arrow indicates the longest PAT thickness on surround in proximal portion of left coronary arteries (LCAs). In this patient, maximal PAT thickness is 21 $\mathrm{mm}$ and this measure was used for analysis.

ing the chi-square test for categorical variables and a Student $t$ test for continuous variables. Values of continuous variables were expressed as mean \pm standard deviation. Pearson correlation analyses were performed
Table 2. Pearson correlations between PAT thickness and clinical parameters

\begin{tabular}{lcc}
\hline \multirow{2}{*}{ Variable } & \multicolumn{2}{c}{ PAT thickness } \\
\cline { 2 - 3 } & $r$ & $p$ value \\
\hline Age & 0.377 & $<0.001$ \\
Body mass index & 0.305 & $<0.001$ \\
Systolic blood pressure & 0.216 & 0.001 \\
Diastolic blood pressure & 0.118 & 0.083 \\
Heart rate & -0.081 & 0.237 \\
Aspartate transaminase & -0.064 & 0.349 \\
Alanine aminotransferase & -0.037 & 0.592 \\
$\gamma$-Glutamyl transferase & -0.075 & 0.329 \\
Total cholesterol & 0.200 & 0.006 \\
Triglyceride & 0.090 & 0.251 \\
Fasting glucose & 0.152 & 0.029 \\
\hline PAT, pericoronary adipose & &
\end{tabular}

PAT, pericoronary adipose tissue.

to assess correlations between PAT thickness and related variables. Logistics regression was used to analyze the independent risk of PAT for HTN after adjusting for clinical variables related to HTN, such as age, BMI, blood pressure, and laboratory findings. A probability 
Table 3. Multiple logistic analyses of hypertension risk factors in patients

\begin{tabular}{|c|c|c|c|c|c|}
\hline \multirow{2}{*}{ Variable } & \multirow{2}{*}{$\mathrm{SE}$} & \multicolumn{2}{|c|}{ Unadjusted } & \multicolumn{2}{|c|}{ Multivariate-adjusted model } \\
\hline & & $p$ value & OR $(95 \% \mathrm{CI})$ & $p$ value & $\mathrm{OR}(95 \% \mathrm{CI})$ \\
\hline PAT & 0.028 & 0.000 & $1.115(1.055-1.178)$ & 0.041 & $1.065(1.003-1.132)$ \\
\hline Age & 0.290 & 0.001 & $2.512(1.424-4.431)$ & 0.016 & $2.193(1.161-4.141)$ \\
\hline BMI in overweight & 0.506 & 0.000 & $6.750(2.505-18.189)$ & 0.001 & $6.077(2.093-17.644)$ \\
\hline Sex & 0.307 & 0.160 & $1.538(0.843-2.806)$ & - & - \\
\hline Diabetes mellitus & 0.345 & 0.129 & $1.568(0.798-3.083)$ & - & - \\
\hline Triglycerides & 0.002 & 0.658 & $1.001(0.997-1.005)$ & - & - \\
\hline Total cholesterol & 0.003 & 0.977 & $1.000(0.994-1.006)$ & - & - \\
\hline AST & 0.003 & 0.397 & $1.003(0.997-1.009)$ & - & - \\
\hline
\end{tabular}

SE, standard error; OR, odds ratio; CI, confidence interval; PAT, pericoronary adipose tissue; BMI, body mass index; AST, aspartate transaminase.

value of $p<0.05$ was the threshold for statistical significance. Statistical analysis was performed using the SPSS version 19.0 (IBM Co., Armonk, NY, USA).

\section{Ethics statement}

This study was deemed exempt from human use review by the Korean National Institute for Bioethics Policy Institutional Review Board and is identified under Po1201509-21-001.

\section{RESULTS}

The mean age of 214 subjects was $63.80 \pm 14.99$ years, and 152 patients (71.0\%) were men. Eighty-one patients (37.9\%) were hypertensive. Baseline demographic, clinical, and biochemical characteristics are presented in Table 1. There were no significant differences in smoking habits, history of diabetes mellitus (DM), AF, liver function tests, lipid profiles, and fasting glucose levels between the groups. The hypertensive patients were characterized by significantly older age $(69.58 \pm 11.69$ years vs. $60.29 \pm 14.98$ years, $p<0.001)$, and a higher BMI $(23.14 \pm$ 3.32 vs. $20.96 \pm 3.28, p<0.001)$. The hypertensive group showed thicker PAT than the normotensive group $(16.30$ $\pm 5.37 \mathrm{~mm}$ vs. $13.06 \pm 5.58 \mathrm{~mm}, p<0.001$ ).

Table 2 presents correlations between maximal PAT thickness and related variables. Pearson correlation analysis showed PAT thickness to be significantly associated with age, BMI, SBP, and total cholesterol. DBP, heart rate, and most laboratory parameters, except for total cholesterol, did not significantly correlate with PAT thickness. Unadjusted odds ratios of HTN were 1.12 (95\% confidence interval [CI], 1.06 to 1.18) with PAT, 2.51 (95\% CI, 1.42 to 4.43 ) with age, and 6.75 (95\% CI, 2.51 to 18.19) with overweight BMI (Table 3). PAT was independently associated with HTN, age, and overweight BMI, even after adjusting for sex, DM, and levels of triglycerides, total cholesterol, and aspartate transaminase (Table 3).

\section{DISCUSSION}

In this study, maximal PAT thickness was found to be positively correlated with age, BMI, SBP, and total cholesterol. The hypertensive group was older, and hypertensive patients had a significantly higher mean PAT thickness, and higher BMI values than normotensive patients. The most relevant information obtained from this study of nonobese patients hospitalized due to noncardiac acute or chronic illness is that PAT thickness is independently associated with HTN, age, and overweight BMI.

Although there were some differences in study populations and methods, results from several studies consistently supported the relationship between EAT and HTN $[10,15,17,18]$. Magnetic resonance imaging was used to show the existence of a link between EAT and HTN, atherosclerosis, and CAD [19]. Computerized tomog- 
raphy findings showed hypertensive patients to have significantly thicker EAT compared to normotensive patients in a study of 127 asymptomatic patients with one or more cardiovascular risk factors [15]. Echocardiographic measurements performed in outpatient clinics indicated that increased EAT thickness may influence circadian patterns of blood pressure, and correlates with an increased prevalence of nocturnal nondipping patterns [18]. Finally, MDCT studies of patients with CAD showed significantly higher mean EAT than those without CAD, and that PAT was significantly correlated with BMI, HTN, and DM history [10]. However, these studies did not characterize nonobese patients who hospitalized due to acute or chronic illnesses.

Several mechanisms have been proposed to explain the association between EAT and HTN. Henrichot et al. [20] suggested that, in metabolic and cardiovascular disease states, PAT expands, becomes hypoxic and dysfunctional, and recruits phagocytic cells. Changes in adipocyte size and increases in macrophage and $\mathrm{T}$ cell infiltration reduce the release of protective adipokines and increase the release of detrimental adipokines such as leptin, resistin, chemerin, vaspin, interleukin-6, or tumor necrosis factor $\alpha$ in the adipose tissue in both, humans and in $o b / o b$ mice [7]. These molecules may directly diffuse into the myocardial tissue and vessel walls, altering the balance between vasodilatory molecules, such as nitric oxide, and vasoconstricting molecules. All of these effects may independently increase blood pressure $[15,21]$.

Although anatomically contiguous, the two components of EAT, PAT and MAT, showed functionally distinct responses in exercising animals [2], wherein MAT showed more improvement of inflammatory response compare to PAT. Studies of human donor heart vessels reported similar findings in PAT, wherein perivascular adipocytes exhibited reduced differentiation and a heightened proinflammatory state compared to subcutaneous adipocytes [22].

However, it remains unknown as to whether PAT exhibits proinflammatory characteristics or related adverse outcomes when compared to MAT in clinical settings. Demircelik et al. [23] found a positive correlation between EAT and obstructive CAD, and PAT was associated with a similar predictive value to that of EAT. In the current study, we measured the PAT, known as having more proinflammatory character in animal and ex vivo study, through a simple and practical method by using MDCT, and demonstrated an association of PAT with HTN even in the population who have noncardiogenic acute or chronic illness. Obesity is related to increased thickness of EAT and PAT [5]. Greenstein et al. [24] suggested that, while PAT may be beneficial in the healthy, lean state by secreting adiponectin and modulating local vascular tone by increasing nitric oxide bioavailability, these benefits are lost in obesity to hypoxia, inflammation and oxidative stress. However, even after excluding obese patients (BMI $\geq 30$ ), PAT was statistically higher in patients with HTN. Dicker et al. [15] also reported increased EAT as a predictor for HTN in an asymptomatic population after excluding obese individuals (BMI > 30). Ultimately, the clinical impact and the interrelationships of obesity, perivascular fat thickness and perivascular fat functions under various conditions, including asymptomatic and acute or chronic illnesses, will require further investigation.

This study has several limitations. First, this is a cross-sectional study without follow-up data with which to explain causal relationships. Second, all subjects were Korean, which may limit the generalization of our findings to other ethnic groups. Third, although some experimental data have suggested PAT to be more proinflammatory than MAT, we did not directly compare the two adipose types. Some studies have considered volumetric measurements of EAT to be more reproducible than those of EAT thickness [5,6]. However, PAT thickness is easier to measure in that it is less time consuming, and PAT has anatomical proximity to the coronary arteries. Although we observed a significant association between PAT thickness and HTN in nonobese populations in acute or chronic illness, further work will be needed to establish the underlying mechanisms responsible for this association.

Maximal PAT thickness is positively correlated with age, BMI, SBP, and total cholesterol. The hypertensive group was older, had a significantly greater PAT thickness, and higher BMI values than the normotensive group. Multivariate analysis showed that age, PAT thickness, and BMI were independent factors associated with HTN in nonobese, hospitalized patients with noncardiac acute or chronic illness. 


\section{KEY MESSAGE}

1. Maximal pericoronary adipose tissue (PAT) thickness was positively correlated with age, body mass index (BMI), systolic blood pressure, and total cholesterol. The most relevant information obtained from this study of nonobese patients hospitalized due to noncardiac acute or chronic illness is that pericoronary adipose tissue thickness is independently associated with hypertension, age, and overweight BMI.

2. Measurement of maximal PAT thickness by using multidetector computed tomography images is easy and practical.

3. Ultimately, the clinical impact and the interrelationships of obesity, perivascular fat thickness and perivascular fat functions under various conditions, including asymptomatic and acute or chronic illnesses, will require further investigation.

\section{Conflict of interest}

No potential conflict of interest relevant to this article was reported.

\section{REFERENCES}

1. Mazurek T, Zhang L, Zalewski A, et al. Human epicardial adipose tissue is a source of inflammatory mediators. Circulation 2003;108:2460-2466.

2. Company JM, Booth FW, Laughlin MH, et al. Epicardial fat gene expression after aerobic exercise training in pigs with coronary atherosclerosis: relationship to visceral and subcutaneous fat. J Appl Physiol (1985) 2010;109:19041912.

3. Bachar GN, Dicker D, Kornowski R, Atar E. Epicardial adipose tissue as a predictor of coronary artery disease in asymptomatic subjects. Am J Cardiol 2012;110:534-538.

4. Szasz T, Webb RC. Perivascular adipose tissue: more than just structural support. Clin Sci (Lond) 2012;122:1-12.

5. Gorter PM, van Lindert AS, de Vos AM, et al. Quantification of epicardial and peri-coronary fat using cardiac computed tomography; reproducibility and relation with obesity and metabolic syndrome in patients suspected of coronary artery disease. Atherosclerosis 2008;197:896-903.

6. Maurovich-Horvat P, Kallianos K, Engel LC, et al. Influence of pericoronary adipose tissue on local coronary atherosclerosis as assessed by a novel MDCT volumetric method. Atherosclerosis 2011;219:151-157.

7. Spiroglou SG, Kostopoulos CG, Varakis JN, Papadaki HH. Adipokines in periaortic and epicardial adipose tissue: differential expression and relation to atherosclerosis. J Atheroscler Thromb 2010;17:115-130.

8. Lim S, Meigs JB. Ectopic fat and cardiometabolic and vascular risk. Int J Cardiol 2013;169:166-176.

9. Thanassoulis G, Massaro JM, Hoffmann U, et al. Prevalence, distribution, and risk factor correlates of high pericardial and intrathoracic fat depots in the Framingham heart study. Circ Cardiovasc Imaging 2010;3:559-566.

10. Aydin AM, Kayali A, Poyraz AK, Aydin K. The relationship between coronary artery disease and pericoronary epicardial adipose tissue thickness. J Int Med Res 2015;43:17-25.

11. Thanassoulis G, Massaro JM, O'Donnell CJ, et al. Pericardial fat is associated with prevalent atrial fibrillation: the Framingham Heart Study. Circ Arrhythm Electrophysiol 2010;3:345-350.

12. Qureshi AI, Suri MF, Kirmani JF, Divani AA, Mohammad Y. Is prehypertension a risk factor for cardiovascular diseases? Stroke 2005;36:1859-1863.

13. Grundy SM, Brewer HB Jr, Cleeman JI, et al. Definition of metabolic syndrome: report of the National Heart, Lung, and Blood Institute/American Heart Association conference on scientific issues related to definition. Circulation 2004;109:433-438.

14. Ertas F, Kaya H, Acet H, et al. Increased echocardiographic epicardial fat thickness is related to impaired diurnal blood pressure profiles. Blood Press 2012;21:202-208.

15. Dicker D, Atar E, Kornowski R, Bachar GN. Increased epicardial adipose tissue thickness as a predictor for hypertension: a cross-sectional observational study. J Clin Hypertens (Greenwich) 2013;15:893-898.

16. World Health Organization. Global data base on body mass index [Internet]. Geneva: World Health Organization, c2016 [cited 2016 Nov 4]. Available from: http://apps. who.int/bmi/index.jsp?introPage=intro_3.html.

17. Sironi AM, Pingitore A, Ghione S, et al. Early hypertension is associated with reduced regional cardiac function, insulin resistance, epicardial, and visceral fat. Hypertension 2008;51:282-288.

18. Sengul C, Cevik C, Ozveren O, et al. Epicardial fat thick- 
ness is associated with non-dipper blood pressure pattern in patients with essential hypertension. Clin Exp Hypertens 2012;34:165-170.

19. Sironi AM, Gastaldelli A, Mari A, et al. Visceral fat in hypertension: influence on insulin resistance and beta-cell function. Hypertension 2004;44:127-133.

20. Henrichot E, Juge-Aubry CE, Pernin A, et al. Production of chemokines by perivascular adipose tissue: a role in the pathogenesis of atherosclerosis? Arterioscler Thromb Vasc Biol 2005;25:2594-2599.

21. Iozzo P. Myocardial, perivascular, and epicardial fat. Diabetes Care 2011;34 Suppl 2:S371-S379.
22. Chatterjee TK, Stoll LL, Denning GM, et al. Proinflammatory phenotype of perivascular adipocytes: influence of high-fat feeding. Circ Res 2009;104:541-549.

23. Demircelik MB, Yilmaz OC, Gurel OM, et al. Epicardial adipose tissue and pericoronary fat thickness measured with 64-multidetector computed tomography: potential predictors of the severity of coronary artery disease. Clinics (Sao Paulo) 2014;69:388-392.

24. Greenstein AS, Khavandi K, Withers SB, et al. Local inflammation and hypoxia abolish the protective anticontractile properties of perivascular fat in obese patients. Circulation 2009;119:1661-1670. 